\title{
SONDE RADIOATTIVE A PERCORSO RIDOTTO
}

\author{
R. Ciatnea - A. Lo Sundo
}

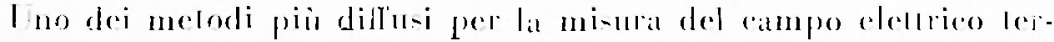
reste e quello delle -onde radioallive. Que-te sono in generale formale da pircoli condultori melallici -ulla rui -uperfere e un deposilo di so-ianzal radioalliva. I na: sondal viene po-la nel punlo in rui si

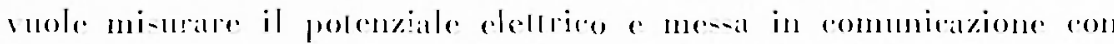
un elellromelos: la so-lanzal radioalliva ionizzal laria rimeo-lanle e quindi tabili-ce le rondizioni perele la -onda arequi-li il potentiale -pellanle allal slal posizione nel camplos.



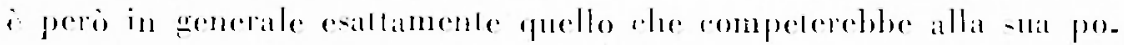
-izione nel rampo. Cio is dovuls primeipalmene al fallo ele learia ionizzala intorns alla -ondal fo-litui-ce in -o-lanzal un eondullore di

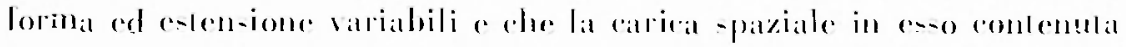
puic aleriale il rampo.

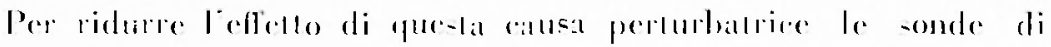

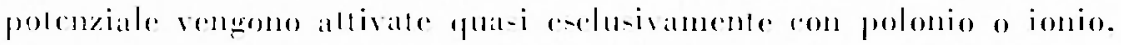



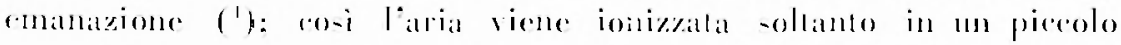

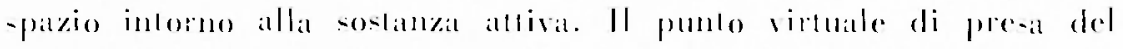
polenziale a quindi pio virino alla -ondar poiche in tal modo -i eli-

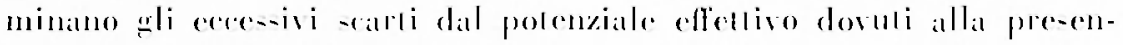
za intorno alla -onda di un nolevole velume di aria ionizzala. Ciò arsiene fluando -i impiegano so-sanze radioallive ele emellono raget

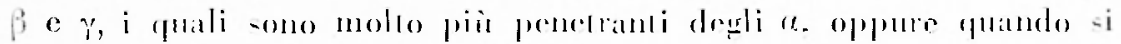

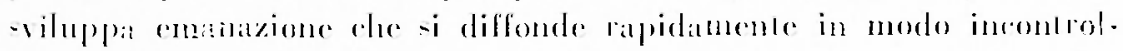
lisbile.

E: levilo pensale quindi che le perturbazioni delle mistre di po-

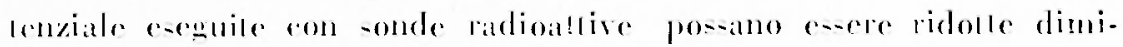

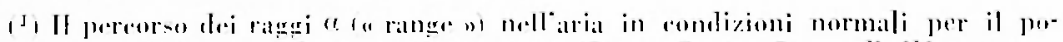

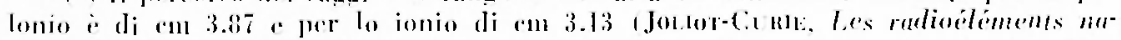
turels. Itermann, Paris, 1916). 
nuendo la lumgheza del peresioo delle parlicelle ionizzanli. Pallen-

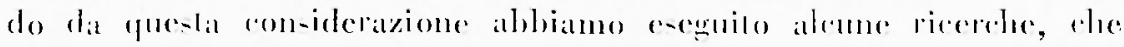
qui coponiamo, allo -eopo di rendere po-ibile ulia mageriore appros-

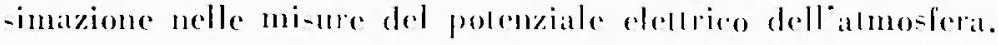

Come i nolo. non si di-pene di sosianze rhe emellano sollanto

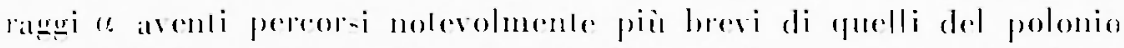
c dello ionio: pertanlo allo ropo di limitare la ionizazione prodolla

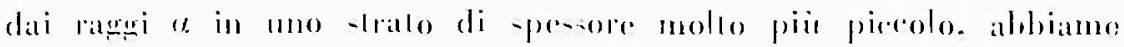

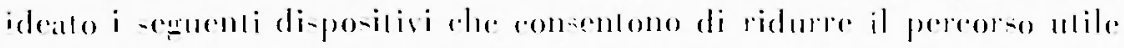
dei rageri uded polonio -les-o.

1) Sonde con ridultore mbolare.

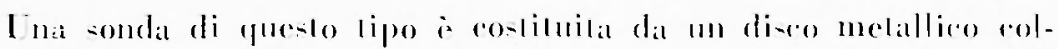

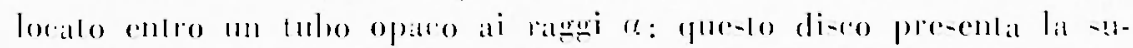

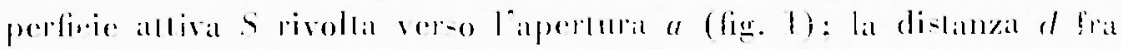

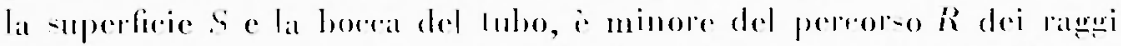
a. Con lale di-posizione, lria-rurando leritel-

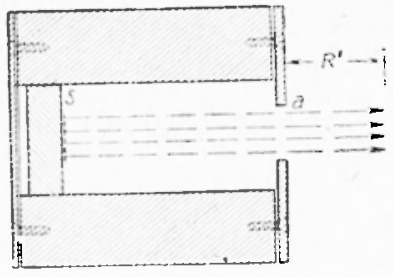

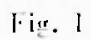
to della diflu-ione degli ioni prodolli nell"in

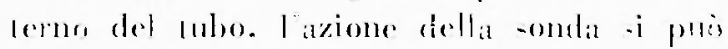

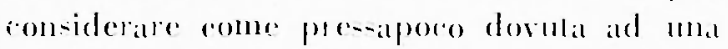
-mperficie allisa romedente ron "apertura "del lubo. emillenle razge " di perrores $R^{\prime}-l=$ dentro un angolo olido più pidolto.

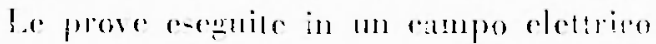
miforme allificials h:1mo mo-lialo. rome -i

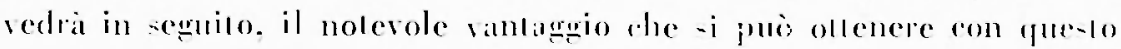
lipo di sonda, specialmente in aria ralma. Si o oservalo pero. rhe il vento può arere ancora mal noterole influenzal -ni valori del polenziale rilevali.

II) Sonde in riduatore ad assorbimento.

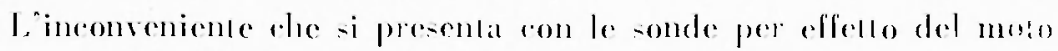
dellaria, ei ha indolto a sperimentare un altro di-po-ilivo, rol qualden

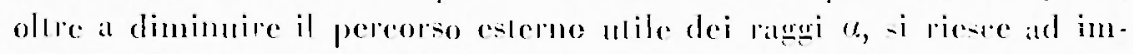
pedire l'intervento degli ioni prodolli nel pereoro inlerno. In que-lo disposilive la relocila dei agari a viene diminuila pel elfello del

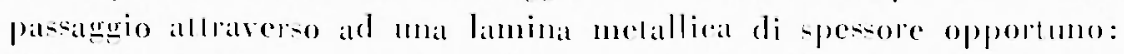

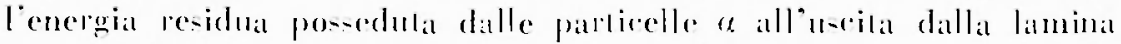
concente la ionizazione dellaria soltane per un pereoro più limilallo. Nelle -onde di fuesto lipo da noi adoperale il rallentamenlo del- 
lo parlicelle a e oftenulo medianle mar foglia di alluminio: lo spese

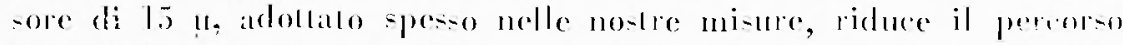

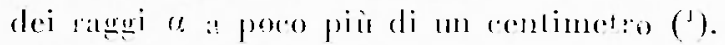

In con-iderazione della l'atgilita della foglia di allominio quarsat viene posta trat due laminetle di


la stperficie in modo ale i ragri e nel loro percoreo. penetrando per i fori del-

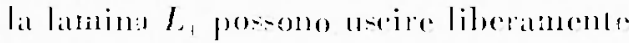
all'eslemo pacindo per i corri-pondenli fori della $L$, dopo avere allaverab!s la lowlia di alluminio 1.

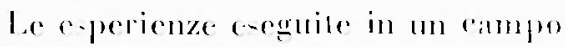
uniforme artifieriale hamno mesio in eri-



Fig. 2 demza, con aria ralma, un comportamento analogूo a quello delle sonde a riduldore lubolare: perio le perturbazioni prodolle dalle correnti di alia sonrs molto minori.

Le sonde venivano -perimentale in an campo molforme crealo an-

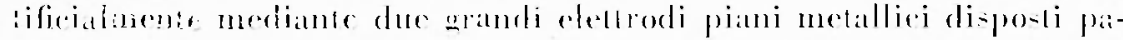

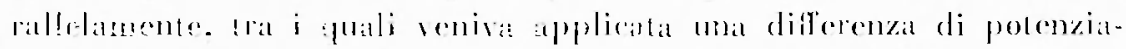

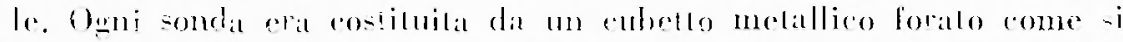

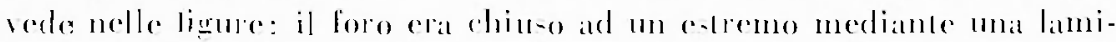

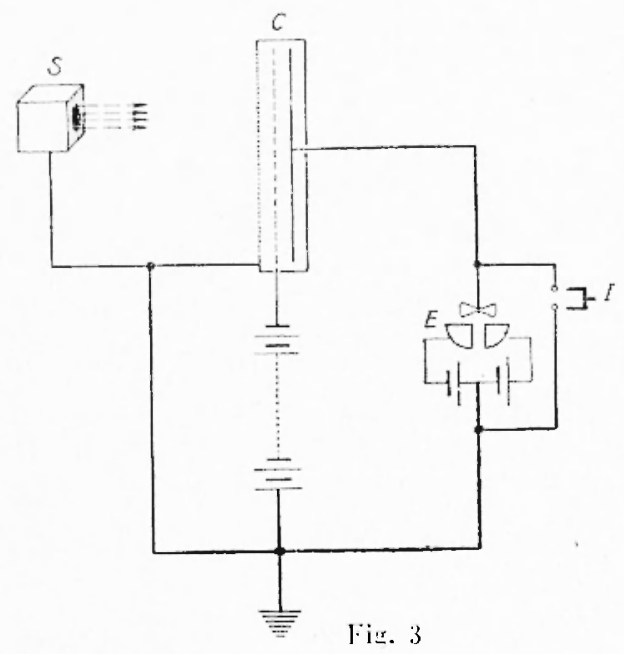
nal melalliciat. c laterialo apere. lo (fig. l) o roperto dial ri. dutlore assorbente. nella par. 10. oppostar (fig. 2). Lim:ieme rra fisealo ad an sollile lubo di olloncenollerato all colellomotro. Questo tubo di sosa pno venivil di-po-lo dentro il rampo parallelamente ai due piani melallici. Nllinlerno del foro era eollorito un pirecolo diseo di ollone argenta. to ed attivalo mediante depo-ilo elentrolition di polo. nia su mal delle due larees. is (figarc l e 2).

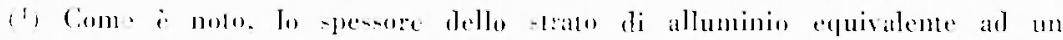

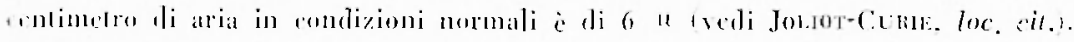




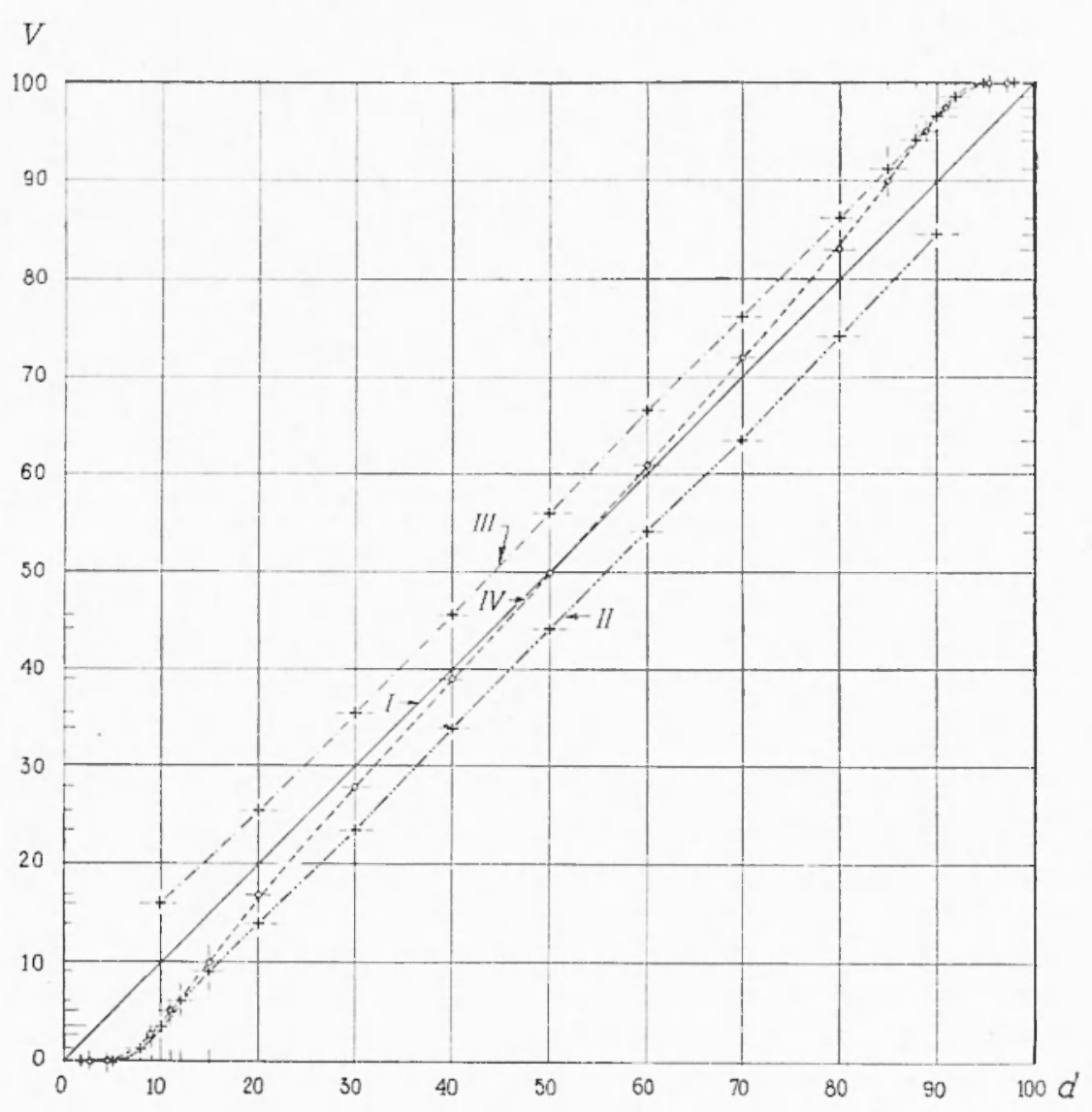

Fig. 4

Le mi-ure ulla lunghezza del percorso utile venivano eseguite mediante una piccola camera a ionizzazione il eui schema è riportato nella fig. 3.

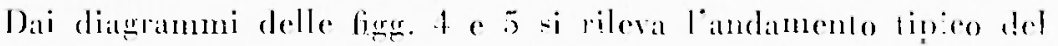
pelemziale di equilibrio a-monto dalle sonde. a pereori normale e ridolli. nei vari punti del rampo elelleireo artifirale da noi adoperato.

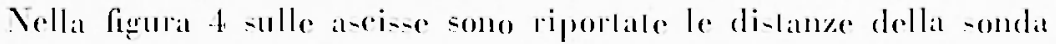
dillearmalura a polenziale zero o -ulle ordinale le relalise dilferenze

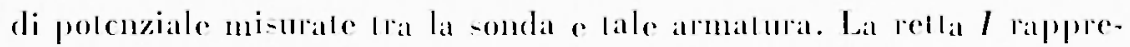
-enla i valori effellivi del polenziale. Le linee $/ 1$ a $/ 1 /$ rappresentimo i vilori del polenziale rilevati mediande ma commerendas a polonio di-posta com la -uperfieie antiva $S$ normale alle linee di forza del 
V

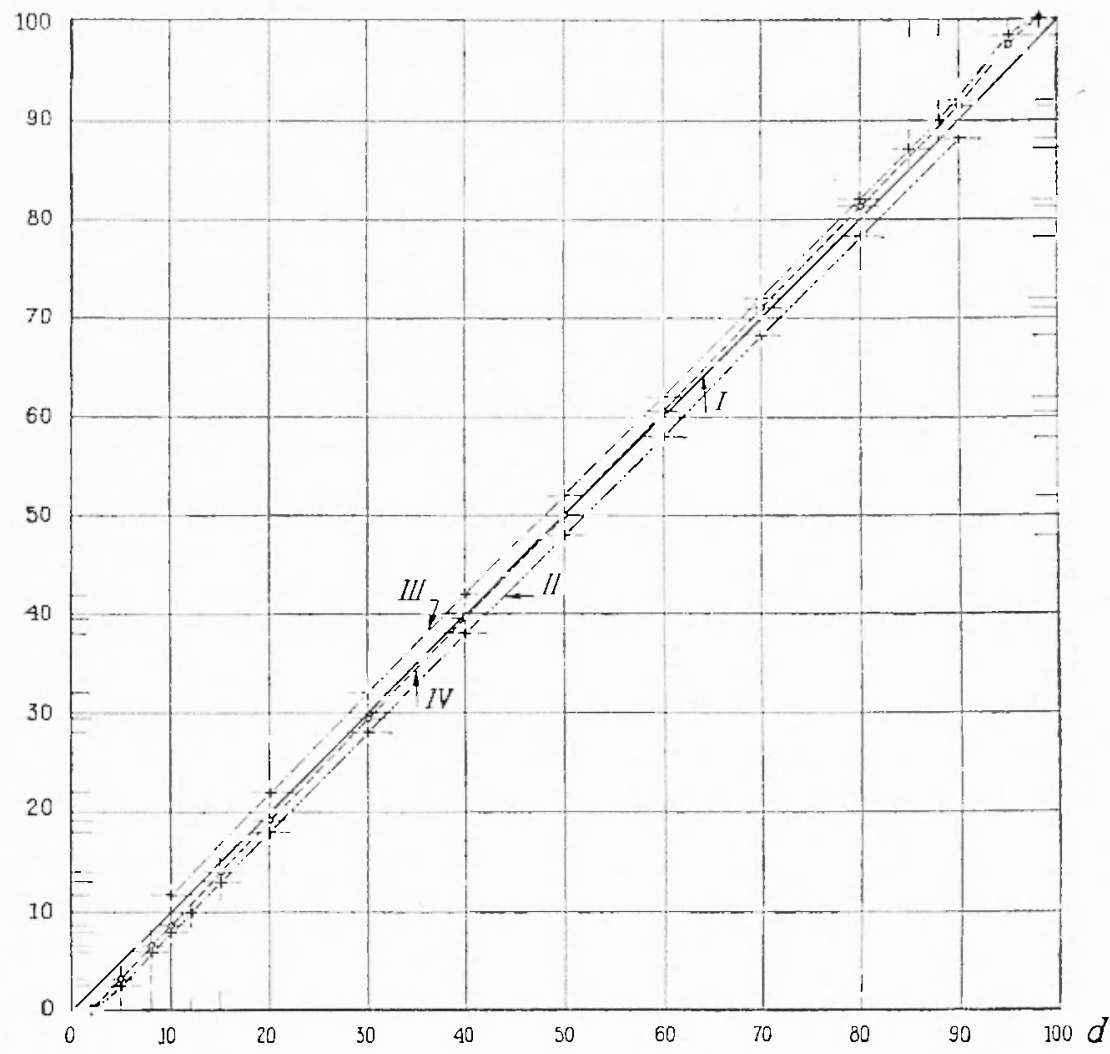

Fig. 5

rampo, e preciantente per i dali rappreentali dalla linea $l l$ la stperfecie allisa reniva rivolta reso larmalumal a potenziale zero, e per la linea $I I I$ rero l'armalura a polenziale 100 . La linea $I T$ rappresen-

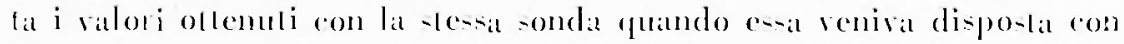
lat -uperfecie allivil parallecla alle linee di lorzat del rampo.

Nella figurat 5 sono riportate mi-ure analoghe esegute con mal -ondir a ridultore tubolare nella quale il pereor-o ulile erat di $12 \mathrm{~mm}$.

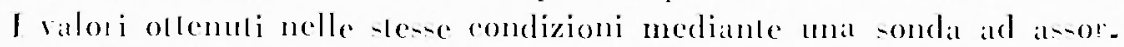
bimento il eni pereoro celerno ulile eril pure di 12 mm, non sono - Lali qui rappresentati perehe approwimaliamente coincidenti eon quelli della sonda a ridullore lubolate.

La figura 6 da approsimativamente un idlea dell"andamento medio della di-lanzat fra il rentro della -onda ed il pumto virtuale ti presa del polenziale. 
In asei-se vengono riporlate. come al solito. le di-tanze deilat -onda dallarmalura a polenziale zero ed in ordinale i valori del rapeporen $\frac{\Delta V}{E}$ dove $\Delta V$ indira la differenzal lra il polenziale mi-nralo quello effellivo. ed $E$ il ramper eleltrieo. Tale rapporlo. ele viene

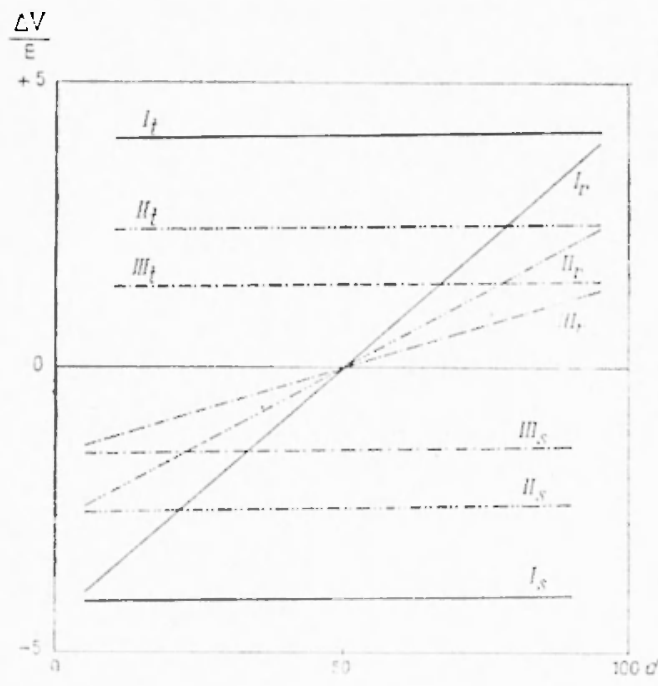

Fiı. 6 eppresen in rin, ri-ulat rome is evidenle, lugrable a!la distanza. lumgono le linee di lorzat, frat il punlo virluale di pre-ia del polemziale ed il antro dellat rondit.

Le linee $i, c$ I, $-i$ riferiseono alle ni-ure litlle ron wnat -ondar a polonio di lipo comume di-po-lat con lat fircie allivat rispellivamenle rivollat rereo latrmallumat al polenziale zero e rerso larmallura a polemziale foll). Lia jincal $I_{r}$ ir relaliva allat - les-al -ondar di-po-lal peró ron la - reperficie alliera parallelia alle linere di lorma del ra!n-

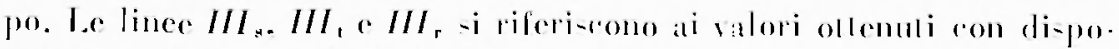
-izioni analoghe medianle la -lesa sonda. munila pero di ridullore ad

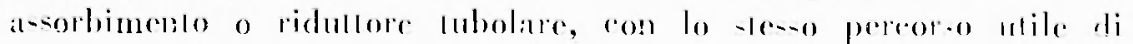
$12 \mathrm{~mm}$, e quindi. come is dello aranti. produrenti in aria ralma lo - lesen effelto (1).

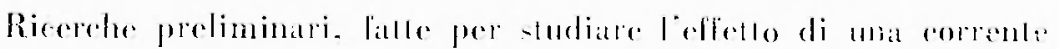

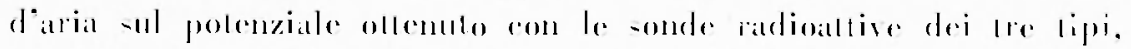


ollenibile nelle misure del polenziale eletrico delloamo-fera. della

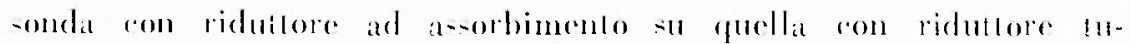

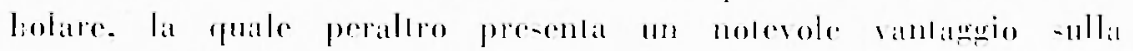
sonda rombune.

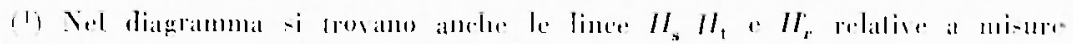

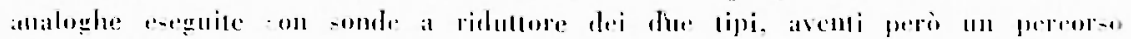
alile di 21 inul. 


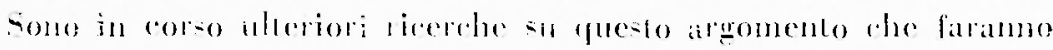
anello di nlla prosimal notal.

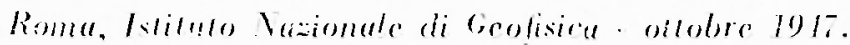

\section{RIASSUTO}

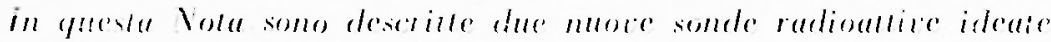

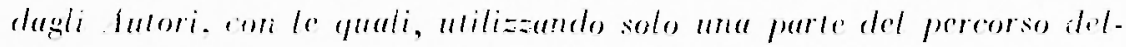

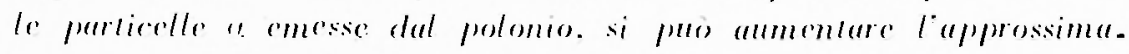
zione nelle misture tel potenziale elebrico dellatmosfora. 